\title{
A New CAC Policy Based on Traffic Characterization in Cellular Networks
}

\author{
Natalia Vassileva, Francisco Barcelo-Arroyo \\ Universitat Politecnica de Catalunya (UPC), \\ c./ Jordi Girona 1-3, Campus Nord, C3 \\ 08034, Barcelona, Spain \\ \{natalia, barcelo\}@entel.upc.edu
}

\begin{abstract}
The Call Admission Control (CAC) method presented in this paper is based on the statistical properties of the network's traffic variables. It probabilistically estimates the time until the release of a seized channel: the admission control depends on the computed mean remaining time averaged along all channels at a specific instant and on a time threshold. The policy produces a smooth transition between the QoS metrics, giving the operator the freedom to design the network at the desired QoS point. Another valuable property is that the algorithm is straightforward and fed only by simple teletraffic metrics: distribution and the first and second moments of Channel Holding Time (CHT). Simplicity is important for a CAC method because decisions for accepting or rejecting calls must be computed quickly and frequently.
\end{abstract}

Keywords: QoS parameters, call admission control (CAC), resource allocation schemes, traffic engineering, wireless cellular systems.

\section{Introduction}

With the increasing number of users and demand for more services in cellular systems, reducing the size of cells is one of the measures typically undertaken to increase the traffic capacity. Smaller cell size leads to more Handovers (HO), and therefore efficient resource allocation mechanisms are required to guarantee the continuation of ongoing calls when Mobile Stations (MS) cross cell boundaries (switch from one Base Station (BS) to another). HO schemes are usually evaluated through two Quality of Service (QoS) parameters: Probability of Blocking $(P B)$ of a new call and Probability of Failure $(P F)$ of handover calls. The former metric evaluates the probability of denying service at the beginning, when a call attempt is received; the latter represents the probability of interrupting a call in progress due to handover drop. In order to bring wireless cellular systems closer to the fixed ones, CAC strategies attempt to reduce $P F$ while maintaining $P B$ at an acceptable level. The Probability of Dropping $(P D)$ a handover is a parameter of interest to the operator, but the user perceives only $P F$ (see Section 4).

This work introduces a new concept into HO schemes - the implementation of a CAC based on the statistical properties of a network's traffic variables. The admission 
control presented here considers the Channel Holding Time (CHT). CHT comprises the time from the instant the channel is assigned to a call (new or handover) until the instant the channel is released. In other words, it is the time spent by the MS in the same cell (i.e., connected to the same BS) while talking. Because of the mobile nature of the MSs, CHT generally differs from the unencumbered call duration. In this work, it is used to probabilistically determine the remaining holding time of the busy channels at a specific instant. This metric can then be used in the design of CAC strategies.

Simplicity is important for a CAC method because the conditions for accepting or rejecting a call must be computed quickly and frequently. Another valuable property is the ability to find the desired balance between $P B$ and $P F$ in order to allow the operator to design the network at the preferred QoS point. As shown below, the CAC introduced in this work is straightforward to compute, is fed only by simple teletraffic metrics, and produces a smooth transition between $P B$ and $P F$.

\subsection{Handover Schemes}

CAC methods prioritize handover traffic before fresh call attempts - HO calls are always admitted as long as there are available resources. Various strategies are used for that purpose. A classic one is the Guard Channel Scheme (GCH) [1], [2], which uses a cutoff policy to reserve a number of resources to be available only to calls in progress. This scheme can include the possibility of a handover to seize a guard channel first, a common channel first, or a probabilistic combinations of both [3]. GCH schemes have only one parameter (the number of guard channels) to tune, and this parameter is an integer value. This makes it difficult to find the desired balance between $P B$ and $P F$. In addition, a general drawback of the cut-off policies is that their use of resources is far from optimal. The Handover Queueing Scheme (HQS) [4] gives priority to $\mathrm{HO}$ calls by permitting them to queue. The attained Carried Traffic (CT) is good, but in general handover calls are too highly prioritized. An extension to these schemes is the Guard Channel with Queue (GCQ) scheme, in which new calls have access to only part of the total capacity and cannot queue.

Fractional Guard Channel (FGC) [1] both exercises finer control of $P B$ and $P F$ probabilities and achieves higher carried traffic than GCS. The Dynamic Guard Channel (DGC) scheme [5] uses the mobility and number of busy channels heuristically in order to allocate free resources to incoming new calls. Other handover priority strategies implement measurement-based parameters, such as transmitted power, time spent in the degradation area, and traffic load in neighbouring cells [3], [4], [6], and [7]. These schemes permit the easy transition between $P B$ and $P F$, but the number of parameters to adjust is so high that these policies are difficult to tune; tuning too many parameters has a high computational cost and makes these schemes impractical [8], [9]. 


\subsection{Goal and Organization}

During analysis of handover schemes, traffic variables are usually assumed to be exponentially distributed to obviate the analytical intractability of the problem. Empirical evaluations [10], [11], [12], and [13] demonstrate that most of the traffic processes that take place in the cellular systems differ from Poisson or negative exponential distribution (n.e.d.). For example, HO arrivals, CHT, dwell time in the overlap area, and other traffic-related random variables (r.v.) are generally not memoryless.

The main goal of this work is to study the applicability of statistical knowledge of the processes observed in cellular systems to the control of QoS parameters. The methodology applied is as follows. The common hypotheses about the holding time are relaxed. At the same time, the assumptions usually made about the arrival process are still present. This allows us to isolate the implications of holding time for CAC functionality and system performance from the implications of the arrival process. The method presented here uses the duration of channel occupancy. The hypothesis for a n.e.d. CHT is relaxed. Based on the time elapsed from seizing a free channel, the remaining time for releasing it can be probabilistically estimated. The estimation of the remaining CHT can be used to compute the mean remaining time for freeing a channel averaged along all the channels. By knowing its value and setting a restrictive or looser time threshold, a different priority can be given to $\mathrm{HO}$ traffic. This provides a window of possible values from which the operator can choose according to the desired trade-off between QoS and carried traffic.

The paper is organized as follows. In Section 2, the analysis of the remaining CHT is reviewed. In Section 3, the HO method is presented. Section 4 outlines the simulation setup, and Section 5 contains the performance figures. Section 5 concludes the paper with summary remarks and directions for future work.

\section{Expected Remaining Time}

The CAC method described in Section 3 implements a metric related to the elapsed channel holding time - the remaining time of a call in service in a channel. We use the terminology introduced in [11] to designate by "remaining time" the time interval between the instant when a decision for a new call acceptance/rejection has to be made and the instant when a resource will become free (i.e., call termination or continuation of the call in a new cell).

The mathematical analysis elaborated and notation used here closely follow those of [11] and [14]; for this reason, only their main results are provided here. According to [14], the remaining time can be estimated by applying the following analysis. It is assumed that a new call can arrive at any instant with equal probability. If $h$ is the remaining time of an ongoing call with a cumulative distribution function (cdf) $F(t)$ and mean service time $m_{1}$, then $h$ is distributed according to the following probability distribution function (pdf): 


$$
f_{h}(t)=\frac{1-F(t)}{m_{1}}
$$

The interest is focused on determining how long the seized resource will remain busy (i.e., in the distribution of the remaining CHT time of an ongoing call) given that the service has already been in progress during time $\varepsilon$. The elapsed time $\varepsilon$ from the beginning of a service is known by the network and can be used to calculate the conditional density of $h, f_{h}(t, \varepsilon$,), in the following way:

$$
f_{h}(t, \varepsilon)=\frac{f(t+\varepsilon)}{1-F(\varepsilon)}=\frac{f(t+\varepsilon)}{1-\int_{0}^{\varepsilon} f(t) d t} .
$$

The average remaining time $\bar{h}(\varepsilon)$ then can be computed from that pdf as:

$$
\bar{h}(\varepsilon)=\int_{0}^{\infty} t f_{h}(t, \varepsilon) d t .
$$

The hypothesis for exponentially distributed CHT was relaxed to permit a holding time with a Square Coefficient of Variation (SCV) different from one. Here, the case of Hyper-Exponential-2 (HE-2) distributed holding time is studied. Some system models differentiate between connections belonging to calls that remain in the same cell and those that require a handover [15], and [16]; others consider two different types of connections (e.g., voice and data). Both cases can be modelled by HE-2 distributions (i.e., by the combination of two n.e.d. r.v. with different means). For simplicity and without loss of generality, a balanced HE-2, in which the time consumed by the two types of n.e.d. combined in the HE-2 is the same, is used in this paper. Erlang-3-distributed CHT $(\mathrm{SCV}=1 / 3)$ is also used in order to study the system for the case of a SCV lower than one. In [10], the Erlang-k distribution is used to fit empirical data for message holding time in cellular systems.

Table 1. Pdf of remaining time $\left(f_{h}(t)\right)$, pdf of remaining versus elapsed time $\left(f_{h}(t, \varepsilon)\right)$, and average remaining time $(h(\varepsilon))$ for Hyper-exponential-2 distributed CHT.

\begin{tabular}{c}
\hline$f_{h}(t)=\frac{1}{m_{1}}\left(p e^{-\mu_{1} t}+(1-p) e^{-\mu_{2} t}\right)$ \\
$f_{h}(t, \varepsilon)=\frac{p \mu_{1} e^{-\mu_{1}(t+\varepsilon)}+(1-p) \mu_{2} e^{-\mu_{2}(t+\varepsilon)}}{p e^{-\mu_{1} \varepsilon}+(1-p) e^{-\mu_{2} \varepsilon}}$ \\
$\bar{h}(\varepsilon)=\frac{1}{\mu_{1}} \frac{p e^{-\mu_{1} \varepsilon}}{p e^{-\mu_{1} \varepsilon}+(1-p) e^{-\mu_{2} \varepsilon}}+\frac{1}{\mu_{2}} \frac{(1-p) e^{-\mu_{2} \varepsilon}}{p e^{-\mu_{1} \varepsilon}+(1-p) e^{-\mu_{2} \varepsilon}}$
\end{tabular}

The algorithm is not limited to specific scenarios. The aforementioned distributions are used to exemplify the principal idea and functionality of the presented CAC method. Concrete distributions for a given system with a different SCV should lead to 
similar qualitative but not quantitative results with a gradual change in the QoS metrics. In Table 1 and Table 2, the main analytical results reviewed are summarized for the used distributions.

For the HE-2-distributed CHT the average remaining time, $\bar{h}(\varepsilon)$, is a monotonically increasing function. The longer the elapsed time, the longer the average remaining. In Table 1, Eq. $4, m_{l}$ is the mean duration of the HE-2 distribution (i.e. $\left.m_{1}=1 / \mu=p / \mu_{1}+(1-p) / \mu_{2}\right)$.

Table 2. Pdf of remaining time $\left(f_{h}(t)\right)$, pdf of remaining versus elapsed time $\left(f_{h}(t, \varepsilon)\right)$, and average remaining time $(h(\varepsilon))$ for Erlang-3.

\begin{tabular}{l}
\hline$f_{h}(t)=\frac{\mu}{3} e^{-\mu t}\left(1+\mu t+\frac{(\mu t)^{2}}{2}\right)$ \\
$f_{h}(t, \varepsilon)=\frac{1}{2} \frac{\mu^{3}(t+\varepsilon)^{2} e^{-\mu t}}{1+\mu \varepsilon+\frac{(\mu \varepsilon)^{2}}{2}}$ \\
$\bar{h}(\varepsilon)=\frac{6+4 \mu \varepsilon+(\mu \varepsilon)^{2}}{2 \mu+2 \mu^{2} \varepsilon+\mu^{3} \varepsilon^{2}}$
\end{tabular}

In contrast to the HE-2, the average remaining time for the Erlang-3 distribution is a decreasing function. The longer the elapsed time of a call during its course, the higher the probability that the channel will soon be released.

\section{System Study with Mean Remaining Time Policy}

The CAC outlined uses simple traffic metrics, such as average CHT, SCV, and number of free/busy channels, as inputs. The latter are readily available, and the first two are easy to compute on-line in the network. They can be estimated along different time windows that must be long enough to allow averaging and short enough to assume that the traffic processes are stationary. When the normal duration of connections and ITU-T recommendations on teletraffic are considered, this window is typically one hour. The distribution of the CHT is assumed to be known in the network. Operating knowing only the first two moments, without a precise knowledge of the distribution, would also be possible, but this task is left for further study.

The algorithm relies on the probabilistically defined remaining CHT of the busy channels. The average remaining time of each busy channel is estimated according to Eq. 3. It is set to zero $(\bar{h}(\varepsilon)=0)$ for the free channels in order to account for currently available resources. A HO call is served as long as there are free channels. In order to determine whether a new call is to be admitted or rejected, the Mean Remaining Time 
$(M R T)$ is averaged along all the channels (i.e., busy and idle; denoted with $C$ in Fig. 1 and formulae) at a specific instant of time, computed as follows:

$$
M R T=\frac{1}{C} \sum_{i=1}^{C} \bar{h}_{i}(\varepsilon)
$$

The ongoing calls are prioritized by setting a Time Threshold (TT). The estimated mean remaining time to release a channel is compared to the time threshold. If a free channel is available and $M R T<T T$, the new call will be admitted to seize it and will not increase the probability of a call interruption $(P F)$, according to the estimated parameter (see Fig. 1). If $M R T>T T$, however, the new call will be rejected in order to admit future $\mathrm{HO}$ arrivals.
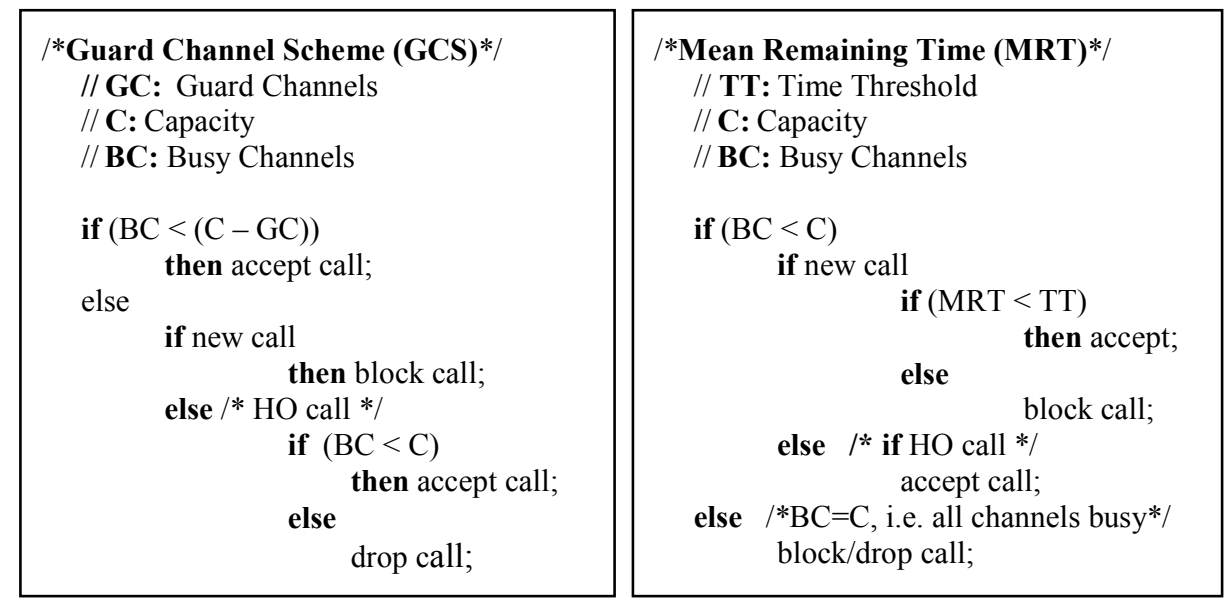

Fig. 1. Guard Channel Scheme (GCS) and Mean Remaining Time (MRT) Scheme.

The GCS can be seen as a particular case of the MRT algorithm for exponentially distributed CHT. Since the estimated residual life of a n.e.d. random variable is equal to its average value independent of the instant of observation [14], the estimation of the remaining CHT does not depend on the elapsed time. All the Busy Channels $(B C)$ will have the same remaining time independent of the elapsed time $\varepsilon$ in each busy channel $j$ :

$$
\bar{h}_{j}(\varepsilon)=\bar{h}=\frac{1}{\mu},
$$

where $1 / \mu$ is the average CHT $\left(m_{l}=1 / \mu\right)$. Independent of the instant of time when $M R T$ is computed, its value will only depend on the number of $B C$, i.e.:

$$
M R T=\frac{1}{C} \cdot \frac{B C}{\mu} .
$$


The correspondence between the two schemes when the CHT is n.e.d. is more clearly seen if the time threshold is set equal to:

$$
T T=\frac{1}{\mu} \cdot \frac{C-G C}{C},
$$

where $G C$ stands for Guard Channels as in Fig. 1. Then, the condition $M R T<T T$ is reduced to that of the GCS (Fig. 1): $B C<C-G C$ ( $C-G C$ is the common pull of channels available to new and $\mathrm{HO}$ calls). Indeed, it is straightforward that, for a particular value of $M R T$ and $T T \in(x-1 ; x)$, where $x$ is a positive integer number, the system response to the admission/rejection of new calls will remain the same and can only change when $T T$ undergoes integer changes. A comparison of the two methods, along with the subtle difference between them, is included in the performance part of the paper.

As analytically demonstrated in Section 2, the elapsed time probabilistically determines the remaining time to release a channel. For the HE-2-distributed CHT, the longer the elapsed time of an ongoing call in a given BS, the longer the expected time it will remain in service (occupying the assigned resource). Thus, the greater the number of channels with an estimated average $h(\varepsilon)$ of high value, the greater the $M R T$ will be. The value of $M R T$ for a given time threshold defines the acceptance or rejection of a new call. If the time threshold is restrictive, the $P B$ for new calls for a particular $T T$ will be elevated for most of the interval of the estimated MRT values. This is the intended logic behind the algorithm: if the state of the busy channels stays unchanged for a long period of time, no more "fresh" calls are to be accepted; otherwise, $\mathrm{HO}$ calls will be rejected and $P F$ will rise correspondingly. If many channels are free and/or the estimated $M R T$ is low, however, "fresh" call requests are to be admitted in the BS to thereby improve $P B$ and efficiently use the system capacity. Bearing in mind the relationship between the elapsed and remaining time (i.e., the longer $\varepsilon$, the smaller the average $h(\varepsilon)$ will be), reciprocal reasoning can be applied to Erlang-3.

\section{Simulation Setup}

In order to obtain the performance metrics of interest, Omnet++ [17] was used to simulate the MRT scheme. Omnet is a modular discrete event network simulator. The teletraffic system was implemented by designing the following modules: Traffic generator (for generating calls - new and HO with a user-defined distribution), Dispatcher (executes the logic of the scheme), Server (for each of the channels), and Statistics (collects the output of the simulation runs; see next subsection on metrics computed and stored in this module). In order to validate the simulator, first the teletraffic system without a HO policy (all calls are accepted as far as there are available resources) and the GCS method were simulated. For these systems, analytical results can be obtained through Markov chains. An excellent agreement between the analytical and simulated results was observed. A similar approach and methodology for studying the performance of traditional HO schemes is applied in [18]. 
Several hypotheses common to other CAC studies (see [19] for example) are assumed in this paper. The wireless system under study is homogenous, and so is the traffic. All cells have the same size and capacity and work under identical traffic conditions (i.e., the arrival intensities of new and $\mathrm{HO}$ calls). The system offers only voice service. As a result, it is sufficient to model and simulate the performance of one cell.

In order to isolate the implications of non-Poisson CHT, the arrival process is assumed to be Poisson with an arrival intensity $\lambda$. This describes Poisson "fresh" and handover incoming traffic with rates $\lambda_{\text {new }}$ and $\lambda_{H O}$, respectively. These metrics are interrelated by the following equations:

$$
\lambda=\lambda_{\text {new }}+\lambda_{H O}, \quad \alpha=\frac{\lambda_{H O}}{\lambda_{\text {new }}},
$$

where $\alpha$ is the mobility factor, which is the ratio of handover to new call arrival rates to the BS (an estimation of mobility). In this paper reported average values for $\alpha$ and the unencumbered call duration $\left(d_{\text {call }}\right)$ are applied, the average duration of the channel holding time $(1 / \mu$ in the formulae and Table 3$)$ can be defined in the following way:

$$
\frac{1}{\mu}=\frac{d_{\text {call }}}{\alpha+1} .
$$

Thus the average CHT is equal to the average call duration divided by the average number of handovers per call plus one; this is the average number of visited cells.

The metrics that the simulation program gives as output values are the number of blocked and served calls. The following formulae are used to compute the performance metrics of interest:

$$
\begin{aligned}
P B & =\frac{\text { Number New Blocked }}{\text { Number New Blocked }+ \text { Number New Served }}, \\
P D & =\frac{\text { Number HO Blocked }}{\text { Number HO Blocked }+ \text { Number HO Served }},
\end{aligned}
$$

where $P D$ is the Probability of Dropping a HO call. $P D$ describes the probability of rejecting a $\mathrm{HO}$ request, whereas $P F$ is the probability that one of the $\mathrm{HO}$ attempts that a call will require along its duration will be dropped and thus will lead to a forced termination of the ongoing call. Since $P D$ cannot be perceived by the users, $P F$ is computed through it. If we take into account that a call on average visits $\alpha+1$ cells (i.e., requires $\alpha \mathrm{HO}$ ), the probability of failure can be computed by [22]:

$$
P F=1-(1-P D)^{\alpha} .
$$

The input parameters and their feeding values are summarized in Table 3. The results shown next are obtained for a mobility of $\alpha=2$. It is in accordance with the reported value in [10]. Note that the average CHT time is defined using Eq. 13 with a reported value for the whole call duration of 120 seconds [10]. 
Table 3. Simulated scenarios.

\begin{tabular}{cccccc}
\hline $\mathrm{A}$ & $\mathrm{C}$ & $\alpha$ & $1 / \mu$ & $\mathrm{SCV}$ & $\mathrm{TT}$ \\
\hline 4.5 and $6.5 \mathrm{Erl}$ & 10 & 2 & $40 \mathrm{~s}$. & 10 and $1 / 3$ & $0-\infty$ \\
\hline
\end{tabular}

\section{Performance Figures}

The performance results in Fig. 2 for light (4.5 Erl - 45\% loaded) and medium (6.5 Erl $-65 \%$ loaded) traffic for the two different CHT distributions demonstrate that the MRT policy smoothly controls the values of the probability of blocking and failure as a function of the time threshold. Since $P B$ and $P F$ are interrelated, the changes they experience are expected. Increasing the $T T$ allows more new calls to enter the system ( $P B$ decreases), whereas it causes $\mathrm{HO}$ calls to become less prioritized ( $P F$ increases).
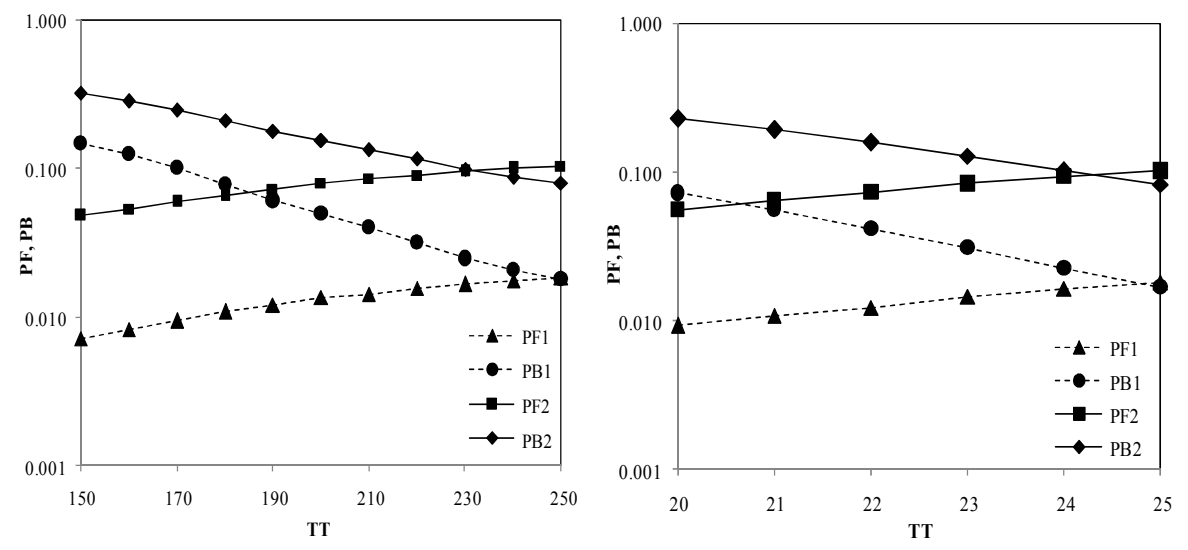

Fig. 2. QoS performance figures for 1) light (45\%) and 2) medium (65\%) traffic load. On the left, HE-2-distributed CHT is shown; on the right, Erlang-3 distributed CHT is shown.

Various simulations were executed for the whole range of possible values of the time threshold under different loads. In Fig. 2, of all the possible values of $P F$ and $P B$, only the interval of practical interest is presented. It is limited by the blocking probability, which is regarded by operators as unacceptable when exceeding $20 \%$. Above a certain point (depending on the distributions and particular values of the CHT: $T T>30$ and $T T>400$ for Erlang- 3 and HE-2 in these scenarios), the $T T$ is so loose that all the traffic is accepted when there are free channels.

Table 4 stores the analytical figures of $P F$ and $P B$ for GCS in order to allow comparison with MRT under the same traffic scenarios. Pure loss (i.e., queueless) teletraffic systems are in general insensitive to the distribution of the holding time [20], and GCS in particular is insensitive to the CHT distribution [21]. Note that the 
first three cases are of practical significance $(P B<20 \%)$ and are QoS points that can be accomplished by the MRT method as well. Therefore, for GCS, the choice of the operator is very limited and restricted to two or three (depending on the traffic load) possible working points. In contrast, it can be observed from the figures that the MRT offers a continuous interval of possible $P B$ and $P F$ pairs. When the load offered to the system increases, the working interval becomes tighter for both schemes. With the MRT, however, there is still a wider operational window compared to the traditional cut-off method. The gradual transition of the blocking and failure probabilities is important, because it gives the operator the freedom to finely adjust the $P F$ and $P B$ figures.

Table 4. Performance figures for the Guard Channel Scheme with 1) $45 \%$ and 2) $65 \%$ traffic load, capacity $C=10$ channels, and $\alpha=2$.

\begin{tabular}{cllll}
\hline Guard Channels & $P B_{1}$ & $P F_{1}$ & $P B_{2}$ & $P F_{2}$ \\
\hline 1 & 0.030 & 0.014 & 0.135 & 0.081 \\
2 & 0.068 & 0.010 & 0.230 & 0.057 \\
3 & 0.132 & 0.007 & 0.347 & 0.041 \\
4 & 0.232 & 0.005 & 0.483 & 0.031 \\
\hline
\end{tabular}

Various performance goals can be set and achieved through the presented CAC method. One possible target value is included in Table 5 along with the corresponding $T T$ value. It shows an interesting case, in which the probability of failure is targeted to be five times smaller than the blocking probability, $P B$ is maintained within reasonable limits (e.g., less then $6 \%$ for HE-2 and around 5.6\% for Erlang-3distributed CHT), and the carried traffic is $4.4 \mathrm{Erl}$ of the attainable $4.45 \mathrm{Erl}$ when no prioritization scheme is implemented.

Table 5. Performance objectives for light load $(A=4.5 \mathrm{Erl})$ and corresponding $T T$.

\begin{tabular}{cccc}
\hline CHT & $T T$ for $(5 \times P F=P B)$ & $P B(\%)$ & $P F(\%)$ \\
\hline HE-2 & 190 & 6.12 & 1.21 \\
Erlang-3 & 21 & 5.62 & 1.08 \\
\hline
\end{tabular}

The Carried Traffic $(C T)$ is a measure of the efficiency of system capacity utilization and is of interest to network operators. It is calculated using the output of the simulation in the following way [22]:

$$
C T=A\left(1-\frac{P B+P F}{\alpha+1}\right) .
$$

With the decrease of $P B$ (increase of $P F$ ), the channel efficiency utilization is increased, since more new calls are admitted and more traffic is carried. The augmentation of the $C T$ increases the operator's revenue, and thus performance figures are usually adjusted according to both the target QoS and $C T$ values - an acceptable tradeoff between quality of service and revenue is sought. In the light of this, Fig. 3 shows the $C T$ for the MRT scheme for medium load. It also displays the 
carried traffic attained by GCS: $6.17 \mathrm{Erl}, 5.98 \mathrm{Erl}$, and $5.73 \mathrm{Erl}$ for the three working points (i.e., for 1, 2, and $3 G C$, Table 4). The MRT scheme performs equally well under the HE-2 and Erlang-3 distributions. Compared to the cut-off scheme with regard to the carried traffic, there are clearly no gains in terms of $C T$. Benefits do exist, however, for the range of possible QoS pairs and $C T$ from which the operator can choose. It can be observed that the operational interval offered by MRT is continuous, which is the main advantage of the algorithm.
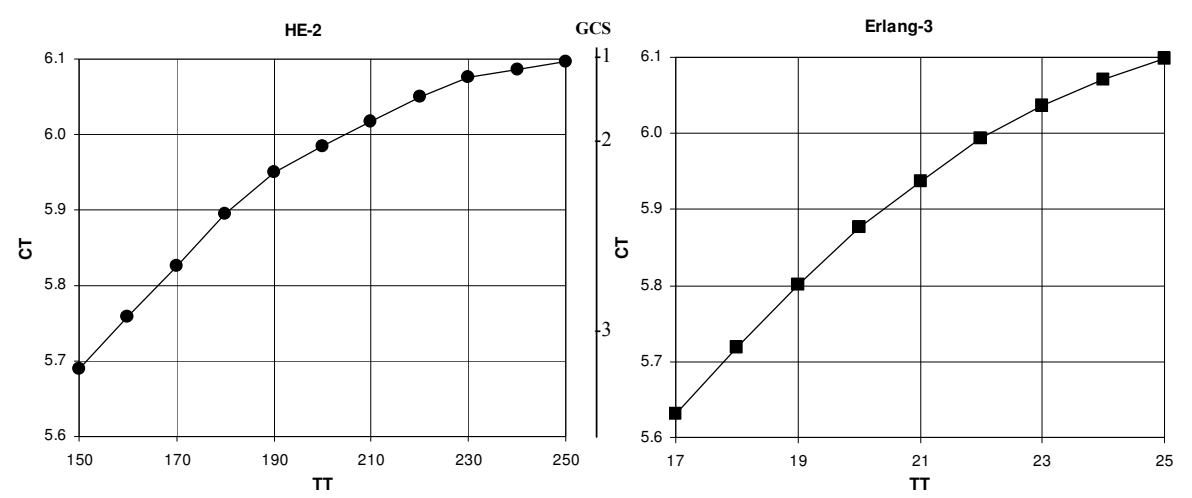

Fig. 3. Carried traffic for a medium (65\%) traffic load using the MRT and GCS method.

\section{Conclusion}

In this work, the application of the expected remaining service time to the design of CAC algorithms was studied and its performance examined via simulation. The results obtained demonstrate that the implementation of CAC with statistical knowledge is advantageous to smoothly regulating the balance between different QoS metrics while still maintaining a high level of simplicity and ease for quick decision computation. When compared to traditional policies like GCS, the MRT method facilitates a wider operational interval. This interval gives the operator the freedom to choose the desired trade-off between QoS and revenue.

This is the first study of CAC with traffic characterization. Further research is required for designing algorithms for scenarios in which the carried traffic is higher but the desired QoS is still met. Also, motivated by the results obtained when relaxing the hypothesis for the holding time distribution, future work will investigate the implications of the arrival process on the design of CAC methods.

Acknowledgments. This research was funded by the Spanish Government and FEDER through the Plan Nacional de I+D (TEC2006-09466/TCM). 


\section{References}

1. Ramjee, R., Nagarajan, R., Towsley, D.: On optimal call admission control in cellular networks. In: IEEE INFOCOM, pp. 45--50 (1996)

2. Yoon, C.H., Un, C.K.: Performance of personal portable radio telephone systems with and without guard channels. IEEE J. Selected Areas Communications, vol. 11, pp. 911--917 (1993)

3. Kulavaratharasha, M.D., Aghvami, A.H.: Teletraffic performance evaluation of microcellular personal communication network (PCNs) with prioritized hand-off procedures. IEEE Trans. Vehicular Technology, vol. 48, pp. 137--52 (1999)

4. Tekinay, S., Jabbari, B.: Handover and channel assignment in mobile cellular networks. IEEE Communications Magazine, vol. 29, pp. 42--46 (1991)

5. Kim, Y.C., Lee, D.E., Lee, B.J., Kim, Y.H., Mukherjee, B.: Dynamic channel reservation based on mobility in wireless ATM networks. IEEE Communications Magazine, vol. 37, pp. 47--51 (1999)

6. Ramanathan, P., Sivalingam, K.M., Agrawal, P., Kishore, S.: Dynamic resource allocation schemes during hand-off for mobile multimedia wireless networks. IEEE J. Selected Areas Communications, vol. 17, pp. 1270--1283 (1999)

7. Agrawal, P., Ankevar, D.K., Narendran, B.: Channel management policies for handovers in celular networks. Bell Labs Technical Journal, pp. 97--110, (1996)

8. Bisaws, S.K, Sengupta, B.: Call admissibility for multirate traffic in wireless ATM networks. In: IEEE INFOCOM, pp. 649--657 (1997)

9. Garcia, D., Martinez, J., Pla, V.: Comparative evaluation of admission control policies in cellular multiservice networks. In: Int. Conf. Wireless Communications pp. 517--531 (2004)

10.Barcelo, F., Jordan, J.: Channel holding time distribution in public telephony systems (PAMR and PCS). IEEE Trans. Vehicular Technology, vol. 49, pp. 1615--625 (2000)

11.Barcelo, F.: Statistical properties of silence gap in public mobile telephony channels with application to data transmission. In: IEEE Int. Conf. Communications (ICC), pp. 2011--2015 (2001)

12.Chlebus, E.: Empirical validation of call holding time distribution in cellular communications systems. In Proc. $15^{\text {th }}$ Int. Teletraffic Congress (ITC), pp. 117--1189 (1997)

13.Jedrzycki, C., Leung, V.C.M.: Probability Distribution of Channel Holding Time in Cellular Telephone Systems. In: IEEE Vehicular Technology Conf. (VTC), pp. 247--51 (1996)

14.Kleinrock, L.: Queueing systems, Volume I: Theory. John Wiley\&Sons (1975)

15.Chih-lin, I., Greenstein, J.L., Gitlin, R.D.: A Microcell/macrocell cellular architecture for low- and high-mobility wireless users. IEEE J. Selected Areas Communications, vol. 11, pp. 885--891 (1993)

16.Steele, R., and Nofal, M.: Teletraffic performance of city street microcells catering for pedestrian mobile users. In: IEE Colloquium on Univ. Research in Mobile Radio (1990)

17.Omnet++ Communite Site, http://www.omnetpp.org

18.Xhafa, A.E., Tonguz, O.K.: Handover performance of priority schemes in cellular networks. IEEE Trans. Vehicular Technology, vol. 57, pp. 565--577 (2008)

19.Hong, D., Rappaport, S.S.: Traffic model and performance analysis for cellular mobile radio telephone systems with prioritized and nonprioritized hand-off procedures. IEEE Trans. Vehicular Technology, vol. VT-35, pp. 77--92 (1986)

20.Iversen, V.: Handbook in Teletraffic Engineering. ITC/ITU-D (2005)

21.Xhafa, A.E., Tonguz, O.K.: Does mixed lognormal channel holding time affect the handover performance of guard channel scheme? In: IEEE GLOBECOM, vol. 6, pp. 3452--3456 (2003)

22.Barcelo, F.: Performance analysis of handoff resource allocation strategies through statedependent rejection scheme. IEEE Trans. on Wireless Communications, no. 3, pp.900--909 (2004) 